\title{
On Controlling Stochastic Sensitivity of Oscillatory Systems
}

\author{
I. A. Bashkirtseva, D. R. Nurmukhametova, and L. B. Ryashko \\ Ural State University, Yekaterinburg, Russia \\ Received July 18, 2012
}

\begin{abstract}
For a nonlinear oscillatory stochastic system, we study the control problem for the variance of random trajectories around a deterministic cycle. To describe the range of random trajectories, we use the method of stochastic sensitivity functions. We consider the problem of designing a given stochastic sensitivity function, discuss problems of controllability and reachability. Complete stochastic controllability is only possible when the control's dimension coincides with the system's dimension. Otherwise, the design problem becomes ill-posed. To solve it, we propose a regularization method that lets us produce a given stochastic sensitivity function with any given precision. The efficiency of the proposed approach is demonstrated with the example of controlling stochastic oscillations in a brusselator model.
\end{abstract}

DOI: $10.1134 / \mathrm{S} 0005117913060040$

\section{INTRODUCTION. PROBLEM SETTING}

Studies of control problems for stochastic systems began in [1]. At present, research results on this topic comprise a rather well developed theory [2-7]. The main emphasis has been put on the control problem on a finite time interval and on constructing controllers that ensure stochastic stability for the equilibrium.

In many applied engineering problems, autooscillations are the fundamental operational modes. Control problems for deterministic oscillatory systems have been considered in a significant number of works [8-10]. Lately, control theory for chaotic oscillations has been developed [11-13].

This work is devoted to controlling stochastically perturbed autooscillations. One needs to account for the necessarily arising random fluctuations in order to understand the dynamics of complex nonlinear systems $[14,15]$.

Development of control methods for established stochastically perturbed modes in dynamical systems presupposes a constructive description of probability distributions for the corresponding stochastic attractors. This distribution is given by the Fokker-Planck-Kolmogorov equation, but it is very hard to find an exact solution for this equation in the control problem we consider for systems of dimension two and beyond.

In the case of small noises, when random states are localized near a deterministic limit cycle, the necessary stationary density can be approximated by a suitable Gaussian distribution whose covariance matrix is given by the stochastic sensitivity function (SSF) [16, 17]. In [18], the SSF method has been extended to cycles in discrete stochastic systems.

This approach has been used to solve the design problem for controllers that form required probability distributions around stable equilibria in both continuous [19] and discrete [20] nonlinear systems. In [21], the resulting methods have been applied to chaos suppression problem.

The purpose of this work is to develop design methods for controllers that produce, based on the SSF technique, given probabilistic characteristics of stochastic autooscillations. 
Let us briefly explain the general approach to solving the stochastic cycle synthesis problem for multidimensional controllable systems of the form

$$
\dot{x}=f(x, u)+\varepsilon \sigma(x, u) \dot{w}(t),
$$

where $x$ is an $n$-dimensional state vector; $u$, an $m$-dimensional control vector; $f(x, u)$, a sufficiently smooth $n$-vector function; $w(t)$, the standard $n$-dimensional Wiener process; $\sigma(x, u)$, a sufficiently smooth $(n \times n)$-matrix function that characterizes how perturbances depend on both state and control; $\varepsilon$, a scalar parameter for the intensity of perturbances.

Suppose that system (1.1) for $\varepsilon=0$ and $u=0$ has a $T$-periodic solution $x=\xi(t)$ with phase trajectory $\Gamma$ (a cycle). We do not assume that cycle $\Gamma$ is stable.

Consider a class of admissible feedbacks $U$ consisting of sufficiently smooth functions $u(x)$ satisfying the following conditions:

$$
\left.u\right|_{\Gamma}=0
$$

and in the closed deterministic system

$$
\dot{x}=f(x, u(x))
$$

solution $x=\xi(t)$ is exponentially orbitally stable in the neighborhood of cycle $\Gamma$.

Condition (1.2) means that function $x=\xi(t)$ remains a solution of system (1.1) for all admissible controls.

The cycle stability problem in the deterministic system (1.3) can be solved with multipliers $\rho_{1}=$ $1, \rho_{2}, \ldots, \rho_{n}$ of the corresponding first approximation system for small deviations $z(t)=x(t)-\xi(t)$ :

$$
d z=(F(t)+B(t) K(t)) z d t
$$

where

$$
F(t)=\frac{\partial f}{\partial x}(\xi(t), 0), \quad B(t)=\frac{\partial f}{\partial u}(\xi(t), 0), \quad K(t)=\frac{\partial u}{\partial x}(\xi(t)) .
$$

A necessary and sufficient condition for exponential orbital stability of cycle $\Gamma$ for the nonlinear system (1.3) is given by the following conditions [22]: $\left|\rho_{i}\right|<1, i=2, \ldots, n$. Multipliers of system (1.4) for fixed $F$ and $B$ are functions of $K: \rho_{i}=\rho_{i}(K)$. Consider the set of $T$-periodic $(m \times n)$ matrices $\mathbb{K}=\left\{K:\left|\rho_{i}(K)\right|<1, i=2, \ldots, n\right\}$. We assume that the set $\mathbb{K}$ is nonempty.

Random trajectories of the stochastic system (1.1) leave the deterministic cycle $\Gamma$ and form around it a stochastic attractor with stationary distribution density $\rho(x, \varepsilon)$.

Function $\rho(x, \varepsilon)$ is a solution of the stationary Fokker-Planck-Kolmogorov equation. Already in the two-dimensional case it becomes a hard problem to analyze this equation. For small noises, in order to approximately find its solution we use the asymptotics

$$
\rho(x, \varepsilon) \approx C \times \exp \left(-\frac{v(x)}{\varepsilon^{2}}\right),
$$

where $v(x)$ is a quasipotential [23] and $C$ is the normalizing factor. To approximate the quasipotential in a small neighborhood of the cycle, the technique of stochastic sensitivity functions has been developed [16].

Let $K \in \mathbb{K}$. We describe the probability distribution of random states of system (1.1) around a stable cycle $\Gamma$ with Poincare sections. Let $\Pi_{t}$ be a hyperplane orthogonal to the cycle at point $\xi(t)$ $(0 \leq t<T)$. For the Poincare section $\Pi_{t}$ in the neighborhood of point $\xi(t)$, we write a quadratic 
approximation of the quasipotential: $v(x) \approx \frac{1}{2}\left(x-\xi(t), W^{+}(t)(x-\xi(t))\right)$. The corresponding Gaussian approximation for the stationary distribution density has the following form:

$$
\rho_{t}(x, \varepsilon) \approx C \times \exp \left(-\frac{(x-\xi(t))^{\top} W^{+}(t)(x-\xi(t))}{2 \varepsilon^{2}}\right) .
$$

The symbol "+" denotes the pseudoinverse. Here the stochastic sensitivity matrix function $W(t)$ for cycle $\Gamma$ due to $K \in \mathbb{K}$ is the unique solution [16] for the Lyapunov equation

$$
\dot{W}=(F(t)+B(t) K(t)) W+W(F(t)+B(t) K(t))^{\top}+P(t) S(t) P(t)
$$

with the following conditions:

$$
\begin{gathered}
W(0)=W(T), \\
W(t) r(t) \equiv 0,
\end{gathered}
$$

where $S(t)=\sigma(\xi(t), 0) \sigma^{\top}(\xi(t), 0), r(t)=f(\xi(t))$, and $P(t)$ is the orthogonal projection matrix onto the hyperplane $\Pi_{t}$. Matrix $\varepsilon^{2} W(t)$ specifies the covariance of random states in the neighborhood of point $\xi(t)$ in the section $\Pi_{t}$.

It is clear that a variation of control $u$ lets us change in Eq. (1.6) only the coefficient $K(t)=$ $\frac{\partial u}{\partial x}(\xi(t))$, so the result of the control, namely the stochastic sensitivity matrix $W(t)$, depends only on the derivative $\frac{\partial u}{\partial x}$. This lets us simplify the controller's structure and, without loss of generality, use in what follows the feedback

$$
u=K(t(x)) \Delta(x)
$$

where $\Delta(x)=x-\gamma(x)$ is the stochastic trajectory's deviation from the deterministic cycle, $\gamma(x)=$ $\operatorname{argmin}_{y \in \Gamma}\|x-y\|, t(x)=\operatorname{argmin}_{t \in[0, T)}\|x-\xi(t)\|$.

To estimate the expenses for control $u$ in the stochastic system (1.1), we will use the function $J_{t}=$ $\mathrm{E}\left(u^{\top}(x) R u(x)\right)$, where the expectation $\mathrm{E}(\cdot)$ is computed with respect to distribution (1.5), and $R$ is a symmetric positive definite $(m \times m)$ matrix. For this cost function, an explicit representation is possible:

$$
J_{t}=\varepsilon^{2} \operatorname{tr}\left(K^{\top}(t) R K(t) W(t)\right) .
$$

The purpose of this control is to form a given stochastic sensitivity function $W$ for the cycle $\Gamma$. The feedback matrix $K(t)$ of controller (1.9) forms in system (1.1) a predefined $T$-periodic stochastic sensitivity matrix $W(t)$; it must, due to (1.6), satisfy the following linear algebraic matrix equation:

$$
\begin{gathered}
B(t) K(t) W(t)+W(t) K^{\top}(t) B^{\top}(t) \\
=\dot{W}-F(t) W(t)-W(t) F^{\top}(t)-P(t) S(t) P(t) .
\end{gathered}
$$

Naturally, a number of problems arise in describing a class of admissible matrices $W(t)$, feasibility conditions for Eq. (1.11), and constructing its solutions and estimating control costs. A detailed study of these problems is given in Section 2 for two-dimensional systems. We will present full controllability conditions under which one can synthesize an arbitrary admissible SSF. In case full controllability is missing the controller design problem becomes ill-posed. We propose a constructive approach to its regularization; it lets us synthesize a given SSF with any given precision.

The efficiency of the proposed approach is demonstrated in Section 3 with the example of solving the controller design problem for a stochastic brusselator model. 


\section{CONTROLLING STOCHASTIC CYCLES OF TWO-DIMENSIONAL SYSTEMS}

In case $n=2$ the projection matrix $P(t)$ can be represented as $P(t)=p(t) p^{\top}(t)$, where $p(t)$ is a unit vector orthogonal to the tangent vector $f(\xi(t))$. Due to condition (1.8), matrix $W(t)$ can be written as $W(t)=\mu(t) P(t)$, where $\mu(t)$ is a $T$-periodic scalar stochastic sensitivity function [17].

The stochastic sensitivity function $\mu(t)$ of cycle $\Gamma$ satisfies the following boundary problem:

$$
\dot{\mu}=a(t) \mu+b(t), \quad \mu(0)=\mu(T),
$$

where

$$
\begin{gathered}
a(t)=p^{\top}(t)\left[(F(t)+B(t) K(t))+(F(t)+B(t) K(t))^{\top}\right] p(t), \\
b(t)=p^{\top}(t) S(t) p(t) .
\end{gathered}
$$

The coefficient $a(t)$ that depends on $K(t)$ can be written as

$$
a(t)=a_{0}(t)+2 \beta^{\top}(t) k(t)
$$

where

$$
\begin{gathered}
a_{0}(t)=p^{\top}(t)\left[F^{\top}(t)+F(t)\right] p(t), \\
\beta(t)=B^{\top}(t) p(t), \quad k(t)=K(t) p(t) .
\end{gathered}
$$

The function $\mu(t)$ gives a detailed description of how stochastic sensitivity changes along a cycle. A convenient characteristic for the cycle's stochastic sensitivity as a whole is the value

$$
m=\max _{t \in[0, T]} \mu(t)
$$

Note that the following explicit representation holds for the multiplier $\rho_{2}[17]$ :

$$
\rho_{2}=\exp \left(\frac{1}{2} \int_{0}^{T} a(t) d t\right)=\exp \left(\frac{1}{2} \int_{0}^{T} a_{0}(t) d t+\int_{0}^{T} \beta^{\top}(t) k(t) d t\right) .
$$

Inequality $\rho_{2}<1$ is a criterion of exponential orbital stability for the deterministic cycle $\Gamma$. This criterion immediately implies two statements.

Statement 1. The set $\mathbb{K}$ of matrices $K(t)$ for controllers (1.9) that stabilize cycle $\Gamma$ of system (1.3) is given by

$$
\int_{0}^{T} \beta^{\top}(t) K(t) p(t) d t<-\frac{1}{2} \int_{0}^{T} a_{0}(t) d t
$$

Statement 2. Suppose that a cycle $\Gamma$ of system (1.3) for $u=0$ is unstable $\left(\int_{0}^{T} a_{0}(t) d t \geq 0\right)$. A necessary and sufficient condition of stabilizability $(\mathbb{K} \neq \emptyset)$ is the condition that $\beta(t) \not \equiv 0$.

Thus, to solve a problem of forming a predefined stochastic sensitivity function $\mu(t)$ for cycle $\Gamma$ of system (1.1) with controller (1.9) it suffices to solve the system of equations

$$
\begin{gathered}
\beta^{\top}(t) k(t)=\frac{\dot{\mu}-a_{0}(t) \mu-b(t)}{2 \mu}, \\
K(t) p(t)=k(t)
\end{gathered}
$$

with respect to the desired matrix $K(t)$ satisfying inequality (2.5).

Here admissible stochastic sensitivity functions comprise the set $\mathbf{M}=\left\{\mu(t) \in C^{1}[0, T] \mid \mu(0)=\right.$ $\mu(T), \mu(t)>0\}$. 
Statement 3. In order for a closed system (1.1), (1.9) to have a given stochastic sensitivity function $\mu(t) \in \mathbf{M}$ it is necessary and sufficient that the feedback matrix $K(t)$ (1.9) satisfies system (2.6), (2.7) and inequality (2.5).

The proof of this statement immediately follows from relations (2.3), (2.4) and Statement 1.

Different cases are possible in solving system (2.6), (2.7).

\subsection{Complete Stochastic Controllability}

Consider Eq. (2.6). The following statement holds.

Statement 4. In order for Eq. (2.6) to be feasible for every function $\mu(t) \in \mathbf{M}$, it is necessary and sufficient that

$$
\forall t \in[0, T] \quad \beta(t) \neq 0 .
$$

Under condition (2.8), for any give $\bar{\mu} \in \mathbf{M}$ system (2.6), (2.7) has an infinite number of solutions, i.e., the controller that forms a given stochastic sensitivity function is not unique. A regularization of this ill-posed problem can be achieved by introducing additional optimization conditions. Let us consider two ways to ensure the uniqueness of this problem's solution.

The first way presupposes an additional formal optimality condition

$$
\|k(t)\|^{2} \longrightarrow \min .
$$

Statement 5. Suppose that condition (2.8) holds. Then a solution of problem (2.6), (2.9) is unique and has the following form:

$$
k(t)=\frac{\alpha(t) \beta(t)}{\beta^{\top}(t) \beta(t)}, \quad \alpha(t)=\frac{\dot{\mu}-a_{0}(t) \mu-b(t)}{2 \mu} .
$$

Proof of this statement follows from the theory of normal pseudosolutions [24].

Substituting the resulting $k(t)$ into (2.7), we get, for the matrix $K(t)$ of controller (1.9), the following equation:

$$
K(t) p(t)=\frac{\alpha(t)}{\beta^{\top}(t) \beta(t)} B^{\top} p(t)
$$

Equation (2.11) is always feasible. For instance, the matrix $K(t)=\frac{\alpha(t)}{\beta^{\top}(t) \beta(t)} B^{\top}(t)$ always provides a solution for it.

If we add the optimality condition

$$
\|K(t)\|^{2} \longrightarrow \min
$$

again, we get a unique solution

$$
K(t)=k(t) p^{\top}(t)
$$

where $k(t)$ satisfies $(2.10)$.

As an alternative way to ensure uniqueness for system (2.6), (2.7), we consider the problem of searching for an optimal control that minimizes the costs (1.10) for synthesizing a given sensitivity function. In the considered case, the cost function satisfies the following representation:

$$
J_{t}=\varepsilon^{2} \mu(t) k^{\top}(t) R k(t) .
$$


Adding to Eq. (2.6) the criterion

$$
J_{t} \longrightarrow \min ,
$$

we pass to a minimization problem whose unique solution has the form

$$
\bar{k}(t)=\frac{\alpha(t) R^{-1} \beta(t)}{\beta^{\top}(t) R^{-1} \beta(t)},
$$

and the corresponding minimal value of the cost function can be found as

$$
\bar{J}_{t}=\frac{\varepsilon^{2} \mu(t) \alpha^{2}(t)}{\beta^{\top}(t) R^{-1} \beta(t)} .
$$

Here $\bar{K}(t)=\bar{k}(t) p^{\top}(t)$.

Thus, given condition (2.8) we can always, with the choice of matrix $K(t)$ for controller (1.9), synthesize in the closed system (1.1), (1.9) any given stochastic sensitivity function $\mu \in \mathbf{M}$. In this case we call the cycle in system (1.1), (1.9) completely stochastically controllable.

In the case of constant matrices $B(t) \equiv B$, condition (2.8) is equivalent to the requirement that $\operatorname{rank}(B)=2$. Thus, for systems with full rank matrices $B$ we have complete stochastic controllability.

In case $\operatorname{rank}(B)=1$, the vector function $\beta(t)$ may turn to zero, which does not let us synthesize certain functions from $\mathbf{M}$. This leads to incomplete stochastic controllability, and in what follows we study this case in detail.

\subsection{Incomplete Stochastic Controllability}

Suppose that there exists a nonempty set $I$ on the interval $[0, T]$ where $\beta(t)$ turns to zero:

$$
\begin{array}{ll}
\forall t \in I & \beta(t)=0, \\
\forall t \in[0, T] \backslash I & \beta(t) \neq 0 .
\end{array}
$$

In this case all reachable stochastic sensitivity functions comprise the set $\mathbf{A}=\{\mu(t) \in \mathbf{M} \mid \forall t \in I$ $\left.\dot{\mu}=a_{0}(t) \mu+b(t)\right\}$.

Statement 6. System (2.6), (2.7) is feasible for every function $\mu(t)$ from the reachability set $\mathbf{A}$. Moreover, the matrix $K(t)$ for the optimal controller that synthesizes $\mu(t) \in \mathbf{A}$ with criterion (2.14) is given by

$$
K(t)= \begin{cases}\frac{\alpha(t) R^{-1} \beta(t) p^{\top}(t)}{\beta^{\top}(t) R^{-1} \beta(t)}, & t \in[0, T] \backslash I \\ 0, & t \in I .\end{cases}
$$

Proof. Feasibility of system (2.6), (2.7) is shown by a direct substitution of the given $K(t)$, while optimality follows from (2.15).

Consider the situation when the desired sensitivity function is not reachable. In this case it is natural to pose the problem of synthesizing a sensitivity function close to the desired one.

Suppose that the desired stochastic sensitivity function $\mu(t)$ is such that on a certain subset $I_{\alpha}$ of the set $I$ the function $\alpha(t)$ does not equal zero. Then Eq. (2.6) is infeasible. This means that not every function $\mu \in \mathbf{M}$ is reachable. Thus, we need to solve an ill-posed problem here: system $(2.6),(2.7)$ is infeasible on the set $I_{\alpha}$, while in other points of the interval $[0, T]$ it has an infinite number of solutions.

In this work, we consider the following regularization method for this ill-posed problem. 
We consider the proposed method for an often encountered case when matrix $B$ is constant and has rank one: $B=b$, where $b$ is a constant two-dimensional vector. Then the scalar function $\beta(t)=b^{\top} p(t)$ turns to zero at those points of the cycle where the tangent to the cycle is parallel to vector $b$. There are at least two such points for every cycle. Let $\beta\left(t_{1,2}\right)=0$ and suppose that at another point of the interval $[0, T]$ it holds that $\beta(t) \neq 0$. We denote by $\bar{\mu}(t)$ the desired stochastic sensitivity function. Equation (2.6) for a scalar function $k(t)$ has the form

$$
\beta(t) k(t)=\alpha(t)=\frac{\dot{\bar{\mu}}-a_{0}(t) \bar{\mu}-b(t)}{2 \bar{\mu}} .
$$

A formal solution $k(t)=\alpha(t) / \beta(t)$ of Eq. (2.17) grows without bound near the points $t_{1}, t_{2}$ for $\alpha\left(t_{1,2}\right) \neq 0$. To avoid the degeneracy related to this unbounded growth of $k(t)$, we perform the following regularization procedure.

Consider small intervals $I_{1}=\left[t_{1}-\delta, t_{1}+\delta\right], I_{2}=\left[t_{2}-\delta, t_{2}+\delta\right]$. The value $\delta$ plays the role of a regularization parameter. On intervals $I_{1}, I_{2}$ we let $k(t) \equiv 0$. Then the dynamics of the real sensitivity function $\mu(t)$ on $I_{1,2}$ does not depend on the control and is given by properties of system (1.1) for $u=0$. The connection of the values $\mu\left(t_{i}-\delta\right)$ and $\mu\left(t_{i}+\delta\right)$ for the function $\mu(t)$ at the ends of interval $I_{i}$ can be found by solving the corresponding Cauchy problems. Indeed, $\mu\left(t_{i}+\delta\right)=m\left(t_{i}+\delta\right)$, where $m(t)$ is a solution of equation

$$
\dot{m}=a_{0}(t) m+b(t)
$$

with initial condition

$$
m\left(t_{i}-\delta\right)=\mu\left(t_{i}-\delta\right) .
$$

We can find an explicit connection between the values $\mu\left(t_{i}-\delta\right)$ and $\mu\left(t_{i}+\delta\right)$ :

$$
\mu\left(t_{i}+\delta\right)=\varphi_{i} \mu\left(t_{i}-\delta\right)+\psi_{i}
$$

where $\varphi_{i}=\varphi\left(t_{i}+\delta\right), \psi_{i}=\psi\left(t_{i}+\delta\right)$, while functions $\varphi(t), \psi(t)$ are solutions of Cauchy problems

$$
\begin{aligned}
\dot{\varphi} & =a_{0}(t) \varphi, & \varphi\left(t_{i}-\delta\right) & =1, \\
\dot{\psi} & =a_{0}(t) \psi+b(t), & \psi\left(t_{i}-\delta\right) & =0
\end{aligned}
$$

and have an explicit representation

$$
\varphi(t)=\exp \left(\int_{t_{i}-\delta}^{t} a_{0}(s) d s\right), \quad \psi(t)=\varphi(t) \int_{t_{i}-\delta}^{t} \frac{b(s)}{\varphi(s)} d s .
$$

On the next step of the regularization procedure we "paste" the values of function $\mu(t)$ on the right end of the interval $I_{i}$ with the value $\bar{\mu}\left(t_{i}+2 \delta\right)$ of the given function $\bar{\mu}(t)$ at point $t_{i}+2 \delta$. To make $\mu(t)$ continuous and avoid discontinuities on the right end of the interval $I_{i}$, we will define function $\mu(t)$ on the interval $\hat{I}_{i}=\left[t_{i}+\delta, t_{i}+2 \delta\right]$ as a linear function $v(t)$ with interpolation conditions

$$
v\left(t_{i}+\delta\right)=\mu\left(t_{i}+\delta\right), \quad v\left(t_{i}+2 \delta\right)=\bar{\mu}\left(t_{i}+2 \delta\right) .
$$

Thus, the reachability set in the class of $\delta$-regularized controls are functions $\mu(t)$ constructed as follows:

(a) on $[0, T] /\left(I_{1} \cup I_{2} \cup \hat{I}_{1} \cup \hat{I}_{2}\right)$ function $\mu(t)$ coincides with the predefined arbitrary function $\bar{\mu}(t) \in \mathbf{M}$. The optimal controller coefficient $K(t)$ corresponding to the given function $\bar{\mu}(t)$ on this segment of the trajectory is given by $K(t)=\frac{\alpha(t)}{\beta(t)} p^{\top}(t)$; 
(b) the value of $\mu(t)$ inside the intervals $I_{1}$ and $I_{2}$ is defined by the solution of Eq. (2.18) with initial conditions (2.19);

(c) on intervals $\hat{I}_{1}, \hat{I}_{2}$ we define $\mu(t)=v(t)$, where $v(t)$ is a linear function constructed with the rule (2.23).

The functions $\mu(t)$ constructed in this way are continuous, and their derivatives may have discontinuities at points $t_{i}+\delta$ and $t_{i}+2 \delta$. The function $\mu(t)$ differs from a given $\bar{\mu}(t)$ only on the set $L=I_{1} \cup I_{2} \cup \hat{I}_{1} \cup \hat{I}_{2}$. For small $\delta$, values of $\mu(t)$ on $L$ are close to the values of the given function $\bar{\mu}(t)$.

\section{EXAMPLE}

Consider a stochastically disturbed brusselator model with the following control:

$$
\begin{array}{ll}
\dot{x}_{1}=f_{1}\left(x_{1}, x_{2}\right)+u+\varepsilon \dot{w}_{1}, & f_{1}\left(x_{1}, x_{2}\right)=a-(b+1) x_{1}+x_{1}^{2} x_{2}, \\
\dot{x}_{2}=f_{2}\left(x_{1}, x_{2}\right)+\varepsilon \dot{w}_{2}, & f_{2}\left(x_{1}, x_{2}\right)=b x_{1}-x_{1}^{2} x_{2},
\end{array}
$$

where $w_{1}, w_{2}$ are independent Wiener processes, $\varepsilon$ is the intensity of random disturbances, and $u$ is the control.

For $u=0$ the corresponding deterministic system $(\varepsilon=0)$ for $b>b^{*}=1+a^{2}$ has a stable limit cycle $\Gamma$ given by a $T$-periodic solution $\left(\xi_{1}(t), \xi_{2}(t)\right)$.

In the presence of stochastic disturbances $(\varepsilon \neq 0)$, random trajectories leave the deterministic cycle and form around it a certain stochastic attractor. Figure 1 shows random trajectories of the stochastic brusselator with parameters $a=0.4, b=1.2, \varepsilon=0.001$ in gray; in black, the corresponding deterministic cycle. Computations have been done with the fourth order Runge-Kutta method with time step $h=0.001$. It is clear that random trajectories fall in a rather nonuniform range around the cycle. The stochastic sensitivity function for this case is plotted on Fig. 2. It is clear that a significant variation of random trajectories around the deterministic cycle on Fig. 1 is caused by large values of the stochastic sensitivity function. Here the stochastic sensitivity coefficient is $m=574$.

Consider the problem of constructing a controller

$$
u\left(x_{1}, x_{2}\right)=k_{1}\left(t\left(x_{1}, x_{2}\right)\right)\left(x_{1}-\xi_{1}\left(t\left(x_{1}, x_{2}\right)\right)\right)+k_{2}\left(t\left(x_{1}, x_{2}\right)\right)\left(x_{2}-\xi_{2}\left(t\left(x_{1}, x_{2}\right)\right)\right),
$$

that would form a predefined stochastic sensitivity function $\bar{\mu}(t) \in \mathbf{M}$ for the brusselator's cycle.

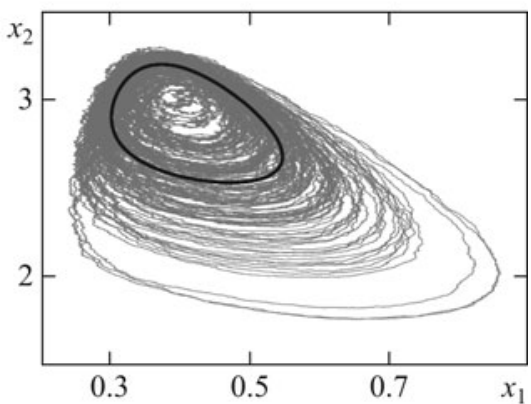

Fig. 1. Random trajectories of a stochastic brusselator with parameters $a=0.4, b=1.2$, $\varepsilon=0.001$ without control (gray); limit cycle of a deterministic brusselator (black).

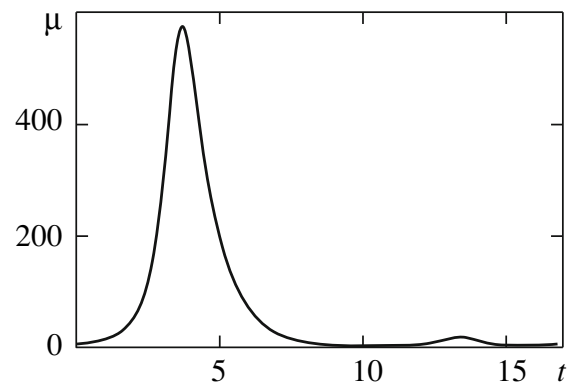

Fig. 2. The stochastic sensitivity function for the brusselator's cycle with $a=0.4, b=1.2$ without control. 
In this example,

$$
B=\left(\begin{array}{l}
1 \\
0
\end{array}\right), \quad p(t)=\frac{1}{\sqrt{f_{1}^{2}(t)+f_{2}^{2}(t)}}\left(\begin{array}{c}
f_{2}(t) \\
-f_{1}(t)
\end{array}\right), \begin{aligned}
& f_{1}(t)=f_{1}\left(\xi_{1}(t), \xi_{2}(t)\right), \\
& f_{2}(t)=f_{2}\left(\xi_{1}(t), \xi_{2}(t)\right) .
\end{aligned}
$$

Since $\operatorname{rank}(B)=1$, the cycle in system (3.1), (3.2) will not be completely stochastically controllable. Indeed, function

$$
\beta(t)=B^{\top} p(t)=\frac{f_{2}(t)}{\sqrt{f_{1}^{2}(t)+f_{2}^{2}(t)}}
$$

turns to zero at points $t_{1}$ and $t_{2}$, where $f_{2}=0$.

In this example, system (2.6), (2.7) that relates parameters $k_{1}(t), k_{2}(t)$ of the controller $(3.2)$ with a given function $\bar{\mu}(t)$ has the following form:

$$
\frac{f_{2}(t)}{\sqrt{f_{1}^{2}(t)+f_{2}^{2}(t)}} k(t)=\alpha(t), \quad \frac{f_{2}(t) k_{1}(t)-f_{1}(t) k_{2}(t)}{\sqrt{f_{1}^{2}(t)+f_{2}^{2}(t)}}=k(t),
$$

where

$$
\alpha(t)=\frac{\dot{\bar{\mu}}(t)-a_{0}(t) \bar{\mu}(t)-b(t)}{2 \bar{\mu}(t)} .
$$

It is clear that this system is degenerate at points $t_{1}$ and $t_{2}$, where $f_{2}=0$.

Let us now apply the regularization method.

We introduce a parameter $\delta$ that characterizes the size of intervals $I_{1}, I_{2}$, neighborhoods of points $t_{1}, t_{2}$. We construct a controller $u_{\delta}$ that ensures $\delta$-reachability for every function $\bar{\mu}(t) \in \mathbf{M}$.

Outside the set $I_{1} \cup I_{2} \cup \hat{I}_{1} \cup \hat{I}_{2}$, coefficients $k_{1}(t), k_{2}(t)$ of controller (3.2) that forms a given function $\bar{\mu}(t)$ satisfy the equation following from system (3.3),

$$
f_{2}^{2}(t) k_{1}(t)-f_{1}(t) f_{2}(t) k_{2}(t)=\alpha(t)\left(f_{1}^{2}(t)+f_{2}^{2}(t)\right) .
$$

Taking as an additional optimality criterion the requirement

$$
k_{1}^{2}(t)+k_{2}^{2}(t) \longrightarrow \min ,
$$

we get a unique solution

$$
k_{1}(t)=\alpha(t), \quad k_{2}(t)=-\alpha(t) \frac{f_{1}(t)}{f_{2}(t)} .
$$

On the trajectory segment corresponding to time moments $t \in I_{i}$ we let $k_{1}(t) \equiv k_{2}(t) \equiv 0$. Thus, it only remains to define the values of $k_{1}(t)$ and $k_{2}(t)$ for $t \in \hat{I}_{i}=\left[t_{i}+\delta, t_{i}+2 \delta\right]$. Here, as before, values of the controller's coefficients are defined by formulas (3.5), in which

$$
\alpha(t)=\frac{\dot{v}(t)-a_{0}(t) v(t)-b(t)}{2 v(t)},
$$

while the linear function $v(t)$ is found by conditions (2.23) and is explicitly given as follows:

$$
\begin{gathered}
v(t)=C_{1} t+C_{2}, \\
C_{1}=\frac{\bar{\mu}\left(t_{i}+2 \delta\right)-\mu\left(t_{i}+\delta\right)}{\delta}, \quad C_{2}=\mu\left(t_{i}+\delta\right)-C_{1}\left(t_{i}+\delta\right),
\end{gathered}
$$

where the value $\mu\left(t_{i}+\delta\right)$ is given by $(2.20)$. 


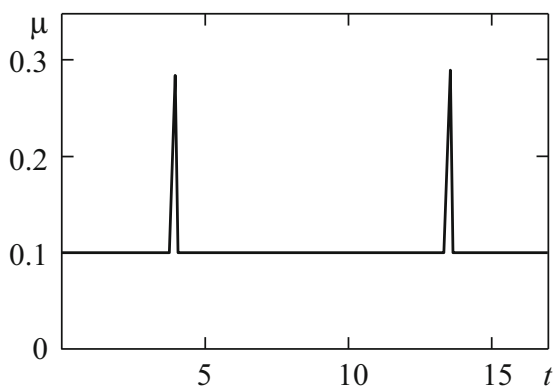

Fig. 3. The stochastic sensitivity function for the brusselator's cycle with $a=0.4, b=1.2$ and controller for $\bar{\mu}=0.1, \delta=0.1$.

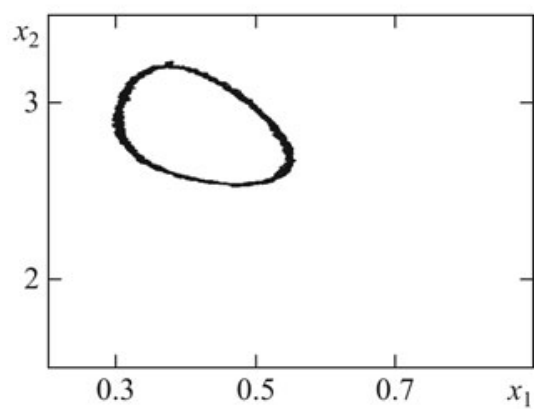

Fig. 4. A stochastic brusselator with parameters $a=0.4, b=1.2, \varepsilon=0.001$ and controller for $\bar{\mu}=0.1, \delta=0.1$.

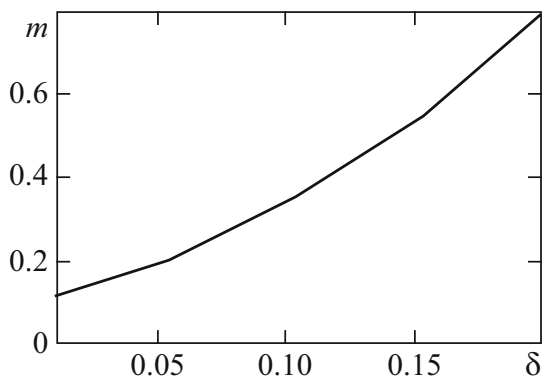

Fig. 5. Dependence of the stochastic sensitivity coefficient for the cycle of the closed system on the regularization parameter.

We take $\bar{\mu} \equiv 0.1$ on the entire interval $[0, T]$. We choose this stochastic sensitivity function because we want to get a bundle of random trajectories with small uniform scatter around the deterministic cycle.

The stochastic sensitivity function of the closed system (3.1) for $a=0.4, b=1.2$ with controller (3.2) corresponding to the designated function $\bar{\mu}$ and parameter $\delta=0.1$ is plotted on Fig. 3. Here the stochastic sensitivity function differs from the designated $\bar{\mu} \equiv 0.1$ on small intervals where it has hits that do not exceed $m=0.29$. It is clear that the resulting control that uses $\delta$-regularization lets us significantly reduce stochastic sensitivity already for $\delta=0.1$ (for a system without control we had $m=574$ ) and keep it at the designated level for the most part of the interval $[0, T]$.

Figure 4 shows the results of direct numerical modeling of random trajectories of the resulting controllable system. Reducing the stochastic sensitivity level has let us localize random trajectories near the deterministic cycle.

The level of hits in the stochastic sensitivity function can be further reduced by reducing the regularization parameter $\delta$ (see Fig. 5).

\section{CONCLUSION}

For a nonlinear oscillatory stochastic system, we have studied the control problem over the stochastic sensitivity function that defined the scatter of random trajectories around a deterministic cycle. For the considered problem of synthesizing a given stochastic sensitivity function, we have discussed problems of controllability and reachability. We have shown that in the absence of complete controllability, the synthesis problem becomes ill-posed and have proposed a regularization 
method that lets us ensure a given SSF with arbitrary precision. Constructive capabilities of the proposed method are demonstrated with the example of solving the controller design problem for a stochastic brusselator model.

\section{ACKNOWLEDGMENTS}

This work was supported by the Ministry of Education and Science of the Russian Federation, project no. 1.1099.2011 and contract no. 14.A18.21.0364.

\section{REFERENCES}

1. Krasovskii, N.N. and Lidskii, E.A., Analytical Design of Controllers in Systems with Random Attributes.. I-III, Autom. Remote Control, 1961, vol. 22, no. 9, pp. 1021-1025; no. 10, pp. 1141-1146; no. 11, pp. 1289-1294.

2. Kurzhanskii, A.B., On Analytic Construction of a Controller in Systems with Noise Dependent on the Control, Differ. Uravn., 1965, vol. 1, no. 2, pp. 204-213.

3. Khas'minskii, R.Z., Ustoichivost' sistem differentsial'nykh uravnenii pri sluchainykh vozmushcheniyakh ikh parametrov (Stability of Systems of Differential Equations under Random Perturbances of Their Parameters), Moscow: Nauka, 1969.

4. Wonham, W.M., Random Differential Equations in Control Theory, New York: Academic, 1970.

5. Fleming, W.H and Rishel, R.W., Deterministic and Stochastic Optimal Control, New York: SpringerVerlag, 1975. Translated under the title Optimal'noe upravlenie determinirovannymi i stokhasticheskimi sistemami, Moscow: Mir, 1978.

6. Kan, Yu.S. and Kibzun, A.I., Stabilization of a Dynamic System which is under the Action of Undetermined and Random Disturbances, Autom. Remote Control, 1990, vol. 51, no. 12, part 1, pp. 1665-1673.

7. Kibzun, A.I. and Sotskii, A.N., Control for a Linear Stochastic System by a Probability Functional, Autom. Remote Control, 1995, vol. 56, no. 5, part 1, pp. 677-683.

8. Chernous'ko, F.L., Akulenko, L.D., and Sokolov, B.N., Upravlenie kolebaniyami (Control of Oscillations), Moscow: Nauka, 1980.

9. Fradkov, A.L. and Pogromsky, A.Yu., Introduction to Control of Oscillations and Chaos, Singapore: World Scientific, 1998.

10. Kovaleva, A.S., Upravlenie kolebatel'nymi i vibroudarnymi sistemami (Control of Oscillatory and Vibroimpact Systems), Moscow: Nauka, 1990.

11. Chaos Control: Theory and Applications, Chen, G. and Yu, X., Eds., Berlin: Springer-Verlag, 2003.

12. Polyak, B.T., Stabilizing Chaos with Predictive Control, Autom. Remote Control, 2005, vol. 66, no. 11, pp. 1791-1804.

13. Fradkov, A.L., Cybernetical Physics: From Control of Chaos to Quantum Control, New York: Springer, 2007.

14. Horsthemke, W. and Lefever, R., Noise-Induced Transitions, Berlin: Springer, 1984.

15. Anishchenko, V.S., Astakhov, V.V., Neiman, A.B., Vadivasova, T.E., et al., Nonlinear Dynamics of Chaotic and Stochastic Systems, Heidelberg: Springer, 2002.

16. Bashkirtseva, I.A. and Ryashko, L.B., Stochastic Sensitivity of 3D-cycles, Math. Comput. Simul., 2004, vol. 66 , pp. 55-67.

17. Bashkirtseva, I.A. and Perevalova, T.V., Analysis of Stochastic Attractors under the Stationary PointCycle Bifurcation, Autom. Remote Control, 2007, vol. 68, no. 10, pp. 1778-1793. 
18. Bashkirtseva, I., Ryashko, L., and Tsvetkov, I., Sensitivity Analysis of Stochastic Equilibria and Cycles for the Discrete Dynamic Systems, Dyn. Contin. Discrete Impuls. Syst., Ser. A: Math. Anal., 2010, vol. 17 , pp. $501-515$.

19. Ryashko, L.B. and Bashkirtseva, I.A., On Control of Stochastic Sensitivity, Autom. Remote Control, 2008 , vol. 69 , no. 7 , pp. 1171-1180.

20. Bashkirtseva, I.A. and Ryashko, L.B., On Stochastic Sensitivity Control in Discrete Systems, Autom. Remote Control, 2010, no. 9, vol. 71, pp. 1833-1848.

21. Bashkirtseva, I., Chen, G., and Ryashko, L., Stochastic Equilibria Control and Chaos Suppression for 3D Systems via Stochastic Sensitivity Synthesis, Commun. Nonlinear Sci. Numer. Simulat., 2012, vol. 17, pp. 3381-3389.

22. Demidovich, B.P., Lektsii po matematicheskoi teorii ustoichivosti (Lectures in Mathematical Theory of Stability), Moscow: Nauka, 1967.

23. Venttsel', A.D. and Freidlin, M.I., Fluktuatsii v dinamicheskikh sistemakh pod deistviem malykh sluchainykh vozmushchenii (Fluctuations in Dynamical Systems under Small Random Disturbances), Moscow: Nauka, 1979.

24. Gantmakher, F.R., Teoriya matrits (Theory of Matrices), Moscow: Nauka, 1966.

This paper was recommended for publication by A.B. Kurzhanskii, a member of the Editorial Board 